\title{
TRANSFERS TO EPIDENDRUM L. FROM OERSTEDELLA RCHB. F.
}

\author{
Eric Hágsater and Miguel A. Soto Arenas
}

Herbario AMO, apdo. postal 53-123, 11320 México D.F., MÉXICO. herbamo@prodigy.net.mx

\begin{abstract}
A cladistic analysis of Epidendrum, its segregates, and of the entire subtribe Laeliinae, based on DNA nucleotidic sequences, has shown that Oerstedella is embedded in Epidendrum. Five new combinations are made and 2 new names are established in order to transfer all Oerstedella species to Epidendrum.

RESUMEN. Un análisis cladístico de Epidendrum y taxa afines, basado en estudios de secuencias de ADN, ha revelado que Oerstedella es un grupo de Epidendrum. Este género tiene tres clados principales: uno mexicano, uno andino y uno ampliamente distribuido. Oerstedella pertenece a este último clado, que es basal en un subclado mayormente centroamericano. Aquí se realizan 5 combinaciones nuevas y se establecen 2 nombres nuevos, para poder transferir todas las especies de Oerstedella a Epidendrum.
\end{abstract}

Key Words / Palabras Clave: Orchidaceae, Epidendrum, Oerstedella.

The genus Oerstedella was established by H.G. Reichenbach (1852) for the Central American Epidendrum centropetalum Rchb.f. Hágsater (May 1981) and Hamer \& Garay (Hamer, October 1981) independently recognized the genus Oerstedella as different from Epidendrum. Oerstedella is distinguished from Epidendrum, with which it shares the fusion of the lip claw to the apex of the column and the presence of a cuniculus-type of nectary, because it has a rostellum perpendicular to the longitudinal axis of the column, which after the removal of the pollinarium is widely sinuate, instead of parallel and slit. Additionally, the lateral lobes of the stigma are not protruding and it lacks a semiliquid viscidium. The presence of a very long clinandrium of the column (in some species) and of warty sheaths clothing the stems (in most species) are also characteristic, although neither one is a synapomorphy of the group. Long clinandria are found in the Physinga group and the Epidendrum albertii Schltr. group of Epidendrum, and warty sheaths may be found, if less conspicuous, in E. propinquum A. Rich. \& Galeotti.

A cladistic analysis of Epidendrum, its segregates (Hágsater \& Soto, in press, Hágsater et al., in prep), and of the entire subtribe Laeliinae (van den Berg et al. 2000, van den Berg 2000), based on nucleotidic sequences of different genomic regions (Internal Transcribed Spacers of nuclear ribosomal DNA, ITS, and the plastid matK and trnL regions) has shown that Oerstedella is embedded in Epidendrum. Epidendrum has three main lineages, a Mexican, an Andean, and a widely distributed clade. Oerstedella belongs to the widely distributed clade and it is basal to a mostly Central American subclade with species bearing distichous inflorescences, including the segregates Auliza (based on Epidendrum ciliare L.), Coilostylis (based on C. emarginata Raf. = E. ciliare L.), Didothion (based on E. clavatum Lindl. $=$ E. purpurascens H.Focke), Epidanthus (based on Epidendrum paranthicus Rchb.f.), Neowilliamsia (based on N. epidendroides Garay), the E. ramosum Jacq. group, and the Spathiger group (based on E. rigidum Jacq.).

Instead of recognizing the twenty-eight segregated genera and proposing some forty new ones in order to make the already existing segregates monophyletic groups, we have decided to recognize only one large, single genus Epidendrum, which is easily set apart in the Laeliinae by the fusion of the column with the lip (though some odd species may have them partially or totally free). Our circumscription of Epidendrum is basically that of Dressler (1984) and Hágsater (1985), except that Oerstedella, Epidanthus, and Neowilliamsia must be included in it. These three taxa have relatively few species compared with the size of Epidendrum (more than 1500 species).

It thus becomes necessary to transfer those Oerstedella taxa for which there are no previous combinations in Epidendrum:

Epidendrum cancanae (P.Ortiz) Hágsater, comb. nova Basionym: Oerstedella cancanae P.Ortiz, Orquideología 22(1): 4. 2001.

TYPE: Colombia: Antioquia, Mpio. Amalfi, Quebrada La Cancana, afluente del Río Porce, 900 m, marzo 2000, L.G. Arango sub P. Ortiz 1121, holo. COL.

Epidendrum fuscinum (Dressler) Hágsater, comb. nova Basionym: Oerstedella fuscina Dressler, Orquídea (Méx.) 8(2): 347. 1982.

TYPE: Panamá: Coclé, comprado en el mercado de 
El Valle de Antón, 29 marzo 1981, R. L. Dressler 6010 , holo. US, iso. AMO!, MO, PMA.

\section{Epidendrum macdougalli (Hágsater) Hágsater, comb. nova}

Basionym: Oerstedella macdougalli Hágsater, Orquídea (Méx.) 13 (1-2): 222. 1994.

TyPe: México: Chiapas, km 2 camino OcozocuautlaLaguna Bélgica, detrás del INI, 900 m, 19 abril 1989, M. A. Soto y E. Martinez 5429, holo. AMO!, clono. AMES!, K!

\section{Epidendrum stolidium Hágsater, nom. nov.}

Based on: Oerstedella ornata Dressler, Orquídea (Méx.) 8(2): 346. 1982.

Type: Panamá: Chiriquí, "La Chumbada" arriba de Guadalupe (cerca del Cerro Punta), 2300 m, 18 octubre 1980, R. L. Dressler 5930, holo. US, iso. AMO!, MO!, PMA, U.

Not Epidendrum ornatum Lem., Fl. des Serres Ser. 1: 4: 334 b. 1848 .

The new name is derived from the Greek $\sigma \tau 0 \lambda \iota \delta$, stolidi, ornament.

\section{Epidendrum parviexasperatum (Hágsater) Hágsater, comb. nova}

Basionym: Oerstedella parviexasperata Hágsater, Orquídea (Méx.) 13 (1-2): 219. 1994.

Type: Costa Rica: San José, Fila de la Cordillera de Talamanca, 1950 m, 2 mayo 1982, E. Hágsater \& C. Horich 6333, holo. AMO!

\section{Epidendrum misasii Hágsater, nom. nov.}

Based on: Oerstedella viridiflora Hágsater, Orquideología 16(2): 192. 1984.

Type: Colombia: Antioquia, Mpio. Valdivia, 21 abril 1983, G. Misas sub E. Hágsater 7234, holo. AMO! Not Epidendrum viridiflorum (Hook.) Lindl., Bot. Reg. Misc. 30, 1842. (=Encyclia viridiflora Hook), nor Epidendrum viridiflorum Sessé \& Mociño, Fl. Mexic. 2nd ed.: 203. 1894.

In honor of Guillermo Misas of Medellín, Colombia who has illustrated numerous orchids for the Chocó region, and provided the pretype material.

\section{Epidendrum $x$ monteverdense (Pupulin \& Hágsater) Hágsater, comb. nova}

Basionym: Oerstedella x monteverdensis Pupulin \& Hágsater, Lankesteriana 8: 32. 2003.
Type: Costa Rica: Puntarenas, Monteverde, Cerro Plano, finca Beeche, $1550 \mathrm{~m}$, col. G. Barboza, cult. Orchid Garden in Monteverde, 2 June 2001, F. Pupulin 3216, holo. USJ.

The following taxa do not need a new combination, because there is an earlier published name for these species available in Epidendrum:

Epidendrum cuneatum Schltr., Repert. Spec. Nov. Regni Veg. 10(81): 456. 1912.

Bolivia: Bei Espíritu Santo, [Río] Antahuacana, ca. 750 m, Juni 1909, O. Buchtien s.n. holo. B, destroyed.

Synonyms: Oerstedella vasquezii Dodson, Icon. Pl. Trop. 6: pl. 563. 1982. Type: Bolivia: Cochabamba, Mpio. Chapare, km 90 [Cochabamba] a Villa Tunari, 1800 m, diciembre 1978, R. Vásquez 80, holo. SEL 36072!, iso. Herb. Vasq.!, LPB, SEL 53394!

Oerstedella thurstoniorum Dodson \& Hágsater, Orquideología 17(3): 139. 1988 ["thurstonorum"]. Type: Ecuador: Hacienda San Antonio Barón von Humboldt, $2.5 \mathrm{~km}$ al norte de Mera, en la carretera de Baños a Puyo, 1050-1300 m, 27 febrero 1985, C. Dodson 15605, holo. QCNE!, iso. MO!, RPSC!

Not Epidendrum thurstoniorum Hágsater, Icon. Orch. (Méx.) 3: pl. 386. 1999 ["thurstonorum"], a member of the $E$. difforme group.

Epidendrum centropetalum Rchb.f., Bot. Zeit. (Berlin) 10(42): 732.1852 (October).

Type: Panamá: Chiriquí, Vulkan, Warszewicz, holo. W! Not E. centropetalum Rchb.f., Bonplandia 3(5): 68. $1855=$ E. viridibrunneum Rchb.f.

Synonyms: Oerstedella centropetala (Rchb.f.) Rchb.f., Bot. Zeit. (Berlin) 10(53): 932. 1852 (December). Oerstedella centradenia Rchb.f., Bot. Zeit. (Berlin) 10(53): 932.1852 (December). Epidendrum centradenia (Rchb.f.) Rchb.f., Walp. Ann. 6: 1163. 1865. Epidendrum tenuiflorum Schltr., Repert. Spec. Nov. Regni Veg. Beih. 3: 49. 1906. Oerstedella tenuiflora (Schltr.) Hágsater, Orquídea (Méx.) 8(1): 24. 1981. Not Epidendrum tenuiflorum hort. ex Lindl., Folia Orch. Epidendrum 88. 1853.

This species has been widely known under the name $O$. centradenia Rchb.f., but $E$. centropetalum Rchb.f. was published two months earlier. A careful revision of the types indicates that they are conspecific. 


\section{REFERENCES}

Dressler, R.L. 1984. The delineation of genera in the Epidendrum complex. Orquídea (Méx.) 9(2): 277298.

Hágsater, E. 1981 (May). Notas sobre Oerstedella: 1. Orquídea (Méx.) 8(1): 19-26.

Hágsater, E. 1985. Towards an understanding of the genus Epidendrum. In: Tan, K. (ed.). Proceedings of the Eleventh World Orchid Conference. Miami, Florida. p. 195-201.

Hágsater, E. \& M.A. Soto Arenas (in press). Epidendrum (generic treatment). In: Pridgeon, A.M., P.J. Cribb, M.W. Chase \& F.N. Rasmussen (eds.). Genera Orchidacearum vol. 4. Epidendroideae (part one). Oxford University Press, Oxford.

Hamer, F. 1981 (October). Las Orquídeas de El Salvador, III: 15, 22, 23, 33. Marie Selby Bot. Gard.
Sarasota, Florida.

Reichenbach, H.G. 1852 (October). Neue Orchideen der Expedition des Herrn J. von Warszewicz. Bot. Zeit. (Berlin) 10 (42): 697-772.

Reichenbach, H.G. 1852 (December). Gartenorchideen V. Bot. Zeit. (Berlin) 10(53): 927-937.

van den Berg, C. 2000. Molecular Phylogenetics of Tribe Epidendreae with Emphasis on Subtribe Laeliinae (Orchidaceae). Ph. D. thesis. University of Reading, England.

van den Berg, C., W.E. Higgins, R.L. Dressler, W.M. Whitten, M.A. Soto Arenas, A. Culham \& M.W. Chase. 2000. A phylogenetic analysis of Laeliinae (Orchidaceae) based on sequence data from Internal Transcribed Spacers (ITS) of nuclear ribosomal DNA. Lindleyana (15): 96-114. 